\title{
Proposta de Protocolo para Redução dos Riscos e Otimização da Sensibilidade nos Exames de Imagem em Pessoas com Diabetes
}

\author{
Proposal for a Guideline of Risk Reduction and \\ Sensitivity Optimization in Imaging Tests for People \\ with Diabetes
}

\author{
Sara DONATO $\triangle^{1}$, Helder SIMÕES ${ }^{1,2}$, Paulo DONATO ${ }^{3,4}$ \\ Acta Med Port 2021 May;34(5):388-394 - https://doi.org/10.20344/amp.15486
}

RESUMO

A diabetes é uma doença crónica muito frequente na população portuguesa, sendo a sua prevalência estimada de $13,6 \%$ nos adultos. A melhor preparação e os riscos associados à realização de exames de imagem em pessoas com diabetes são frequentemente objeto de dúvidas. Neste artigo, pretendemos rever as principais precauções e sugerir um protocolo de atuação, de forma a reduzir os riscos e otimizar a sensibilidade destes exames nesta população. Revemos também a necessidade de suspensão de metformina após a administração de contraste iodado endovascular, pelo risco de acidose láctica, as precauções em pessoas com diabetes insulinotratadas ou sob fármacos que apresentam maior risco de hipoglicemia na realização de exames de imagem que obriguem a jejum, e a influência da hiperglicemia e da terapêutica anti-diabética na sensibilidade da PET-FDG. Com a revisão deste tema e apresentação de um protocolo de atuação pretendemos desmistificar a orientação dos indivíduos com diabetes que vão ser submetidos a exames de imagem, tornando mais simples a sua gestão.

Palavras-chave: Diabetes Mellitus Tipo 1; Diabetes Mellitus Tipo 2; Diagnóstico por Imagem; Meios de Contraste; Tomografia com Emissão de Positrões

\section{ABSTRACT}

Diabetes is a very common chronic disease in the Portuguese population, with an estimated prevalence of $13.6 \%$ in the adults. Doubts often arise regarding the best preparation and the risks associated with doing imaging tests in these patients. In this article we intend to review the main precautions in imaging tests in people with diabetes and to suggest a guideline to reduce the risks and optimize the sensitivity of these tests in this population. The main topics addressed in this article are the need to suspend metformin after the administration of endovascular iodinated contrast due to the risk of lactic acidosis, the precautions in insulin-treated patients or those taking medicines with a higher risk of hypoglycemia when performing imaging tests that require fasting, and the influence of hyperglycemia and of anti-diabetic therapy on the sensitivity of PET-FDG. With this review and the presentation of a guideline, we intend to demystify and simplify the management of individuals with diabetes who are undergoing imaging tests.

Keywords: Contrast Media; Diabetes Mellitus, Type 1; Diabetes Mellitus, Type 2; Diagnostic Imaging; Positron-Emission Tomography

\section{INTRODUÇÃO}

A diabetes é uma doença crónica cuja prevalência está a aumentar em todo o mundo. ${ }^{1}$ Os últimos dados do Observatório Nacional da Diabetes apontam para uma prevalência na população portuguesa de $13,6 \%$ de indivíduos com diabetes e de $28,0 \%$ com pré-diabetes entre os 20 e os 79 anos. $^{2}$

A utilização dos exames de imagem nestes doentes, à semelhança do que acontece na população geral, também tem sido crescente. ${ }^{3,4}$ Frequentemente surgem dúvidas quanto à melhor preparação e quanto aos riscos associados à realização dos mesmos, em particular quando se administra contraste radiológico iodado endovascular.

Os exames de imagem que com maior frequência colocam problemas na pessoa com diabetes são: 1) os que envolvem a administração de contraste iodado endovascular (tomografia computorizada (TC) e angiografia), uma vez que podem provocar lesão renal aguda (LRA) associada ao contraste e propiciar acidose metabólica associada à utilização de metformina; 2) exames que obriguema jejum em indivíduos que estejam sob terapêuticas com risco de hipoglicemia, uma vez que podem aumentar o risco deste efeito secundário; 3) a tomografia com emissão de positrões (PET) com 18F-fluorodesoxiglicose (FDG), na qual tanto a hiperglicemia como algumas terapêuticas (metformina e insulina) podem diminuir a sensibilidade do exame.

Cerca de $90 \%$ das pessoas com diabetes têm diabetes tipo 2 (DM2), que se encontra associada ao excesso de peso/obesidade e envelhecimento. ${ }^{5}$ Esta é provocada fundamentalmente por uma resistência periférica à ação da insulina associada a disfunção da célula $\beta$ pancreática. ${ }^{5,6}$ A prevalência estimada de diabetes tipo 1 (DM1) na Europa é de 2,12/10 000 habitantes e encontra-se também a

1. Serviço de Endocrinologia. Instituto Português de Oncologia de Lisboa Francisco Gentil. Lisboa. Portugal.

2. Departamento de Endocrinologia. Nova Medical School. Faculdade de Ciências Médicas. Universidade Nova de Lisboa. Lisboa. Portugal.

3. Serviço de Imagem Médica. Centro Hospitalar e Universitário de Coimbra. Coimbra. Portugal.

4. Clinica Universitária de Radiologia. Faculdade de Medicina. Universidade de Coimbra. Coimbra. Portugal.

$\triangle$ Autor correspondente: Sara Donato. saramldonato@gmail.com

Recebido: 10 de dezembro de 2020 - Aceite: 19 de fevereiro de 2021 - First published: 31 de março de 2021 - Online issue published: 03 de maio de 2021

Copyright $\odot$ Ordem dos Médicos 2021 
Tabela 1 - Gestão da metformina de acordo com os níveis de TFGe

\begin{tabular}{lcc}
\hline TFGe & Dose máxima & Recomendações \\
\hline$>60 \mathrm{~mL} / \mathrm{min} / 1,73 \mathrm{~m}^{2}$ & $2550 \mathrm{mg} /$ dia & \\
$45-60 \mathrm{~mL} / \mathrm{min} / 1,73 \mathrm{~m}^{2}$ & $2000 \mathrm{mg} / \mathrm{dia}$ & \\
$30-45 \mathrm{~mL} / \mathrm{min} / 1,73 \mathrm{~m}^{2}$ & $1000 \mathrm{mg} /$ dia & Não deve ser iniciada \\
$<30 \mathrm{~mL} / \mathrm{min} / 1,73 \mathrm{~m}^{2}$ & - & Deve ser suspensa \\
\hline
\end{tabular}

aumentar. ${ }^{7}$ Está relacionada com um défice de produção pancreática de insulina e o seu tratamento exige administração de insulina exógena. ${ }^{7,8}$

A metformina pertence à classe das biguanidas e é considerada de primeira linha na terapêutica farmacológica da DM2.5,8 O seu mecanismo de ação não se encontra totalmente conhecido, mas leva a otimização da sensibilidade à insulina a nível hepático e a redução da insulinorresistência periférica. ${ }^{5,8}$ Os efeitos secundários são mais comuns no início da terapêutica e podem ser minimizados pela introdução gradual do fármaco. ${ }^{5,8}$ Os mais frequentes são náuseas, diarreia e dor abdominal. ${ }^{8}$

Esta terapêutica é frequentemente utilizada na prática clínica, não só na DM2, mas também na diabetes secundária a corticoterapia, diabetes pós-transplante, diabetes gestacional, pré-diabetes, síndrome do ovário poliquístico e até, por vezes, na DM1..$^{8-10}$ Em 2018 em Portugal, venderam-se cerca de 3 milhões de embalagens de metformina no ambulatório. ${ }^{2}$ Devido à sua eficácia, baixo custo e à sua boa tolerância em geral é facilmente perceptível a razão pela qual isto acontece. ${ }^{8}$

A metformina não é metabolizada e a sua excreção é feita apenas por via renal, pelo que a dose máxima deve ser ajustada à taxa de filtração glomerular estimada (TFGe). ${ }^{8,11,12} \mathrm{Na}$ Tabela 1 apresentam-se as recomendações de gestão da metformina de acordo com o valor de TFGe. . $, 8,11,12$

A doença renal diabética acomete $20 \%$ - $40 \%$ das pessoas diabéticas. Geralmente surge 10 anos após o início da doença na DM1 e pode já estar presente ao diagnóstico na DM2. ${ }^{11}$

A insulinoterapia é uma terapêutica muito utilizada para o tratamento de pessoas com diabetes, não só no tipo 1 , mas também nas formas de diabetes secundárias, na DM2 de longa evolução onde já existe insulinopenia e na diabetes gestacional. $5,8,9$

Neste artigo pretendemos rever quais as principais precauções nos exames de imagem em pessoas com diabetes e sugerir um protocolo de atuação de forma a reduzir os riscos e otimizar a sensibilidade destes exames nesta população.

\section{Lesão renal aguda associada ao contraste}

A lesão renal aguda (LRA) associada ao contraste é a principal causa iatrogénica de LRA. ${ }^{13,14} \mathrm{O}$ seu risco prende-se sobretudo com a utilização de contraste iodado, uma vez que o gadolínio, nas doses aprovadas para utilização em ressonância magnética, não se parece associar. ${ }^{13,14}$

Embora não exista nenhum procedimento particular em pessoas com diabetes, a utilização de gadolínio em doentes com doença renal crónica avançada ou lesão renal aguda pode-se associar a eventual fibrose sistémica nefrogénica. ${ }^{15}$

A administração endovascular de contraste iodado é utilizada nos estudos por TC e angiografia. ${ }^{13}$ Destes, os exames mais frequentemente realizados são as TC, tendo-se realizado no ano de 2018 em Portugal, 205 por cada 1000 habitantes em ambiente hospitalar. ${ }^{16}$ Neste tipo de exames é usado contraste intra-venoso, enquanto nas angiografias pode ser usado contraste intra-venoso ou intra-arterial. ${ }^{13}$

A definição de LRA pós-contraste não é uniforme entre os estudos, o que torna difícil comparar a incidência de efeitos adversos. ${ }^{13,14}$ No entanto, a definição mais consensual é um agravamento da creatinina maior ou igual a 0,3 $\mathrm{mg} / \mathrm{dL}(26,5 \mu \mathrm{mol} / \mathrm{L})$ ou maior ou igual a 1,5 vezes o valor basal, 48 a 72 horas após a administração de contraste intravascular. ${ }^{13,14,17}$

A fisiopatologia da LRA associada ao contraste não se encontra ainda totalmente conhecida. No entanto, parece haver contribuição simultânea de três mecanismos básicos: vasoconstrição renal e hipóxia medular, toxicidade celular tubular, e formação de espécies reativas de oxigénio. Estas alterações culminam em apoptose celular endotelial e epitelial e diminuição da TFG. ${ }^{18}$

$\mathrm{Na}$ Tabela 2 encontram-se os fatores de risco para ocorrência de LRA pós-contraste..$^{13,17}$

É considerado contraste intra-arterial com primeira passagem renal aquele que chega às artérias renais relativamente não diluido (administração de contraste no coração esquerdo, aorta torácica e supra-renal ou artérias renais), e contraste intra-arterial com segunda passagem renal o contraste que chega às artérias renais após diluição na circulação periférica e pulmonar (administração contraste no coração direito, artérias pulmonares, carótidas, subclávias,

Tabela 2 - Fatores de risco associados a LRA pós-contraste ${ }^{13}$

\footnotetext{
Fatores de risco para LRA pós-contraste:

LRA conhecida ou suspeita

Dose alta de contraste intra-arterial com primeira passagem renal

Contraste com elevada osmolalidade

Múltiplas administrações de contraste nas úlitmas $48-72$ horas

UCl: unidade de cuidados intensivos
}

TFGe $<45 \mathrm{~mL} / \mathrm{min} / 1,73 \mathrm{~m}^{2}$ se contraste intra-arterial com primeira passagem renal ou doente em $\mathrm{UCl}$

$\mathrm{TFGe}<30 \mathrm{~mL} / \mathrm{min} / 1,73 \mathrm{~m}^{2}$ se contraste intra-venoso ou intra-arterial com segunda passagem renal 
coronárias, mesentéricas ou infra-renais). ${ }^{13,17}$

A LRA pós-contraste é potencialmente prevenível, e existem várias recomendações na literatura sobre como reduzir a sua incidência. ${ }^{13,14,17} \mathrm{Em}$ doentes com doença renal crónica, a utilização de fármacos potencialmente nefrotóxicos (ex: anti-inflamatórios não esteroides, inibidores seletivos da COX-2), fármacos anti-microbianos (ex: gentamicina), ou agentes de quimioterapia (ex: cisplatina), deve ser minimizada sempre que possível antes da realização do contraste iodado. ${ }^{13}$ No entanto, esta interrupção pode levar a descompensação da doença de base e à sua suspensão permanente pelo doente..$^{13}$

Baseados em vários ensaios randomizados e controlados a European Society of Urogenital Radiology recomenda que os doentes com fatores de risco para LRA pós-contraste façam profilaxia com hidratação endovenosa antes da administração de contraste iodado. ${ }^{13}$ Esta profilaxia depende do tipo de contraste que vai ser administrado, e encontra-se descrita na Tabela $3 .^{13}$

A evidência atual sugere que a eficácia dos protocolos com infusão salina e com bicarbonato seja semelhante na profilaxia de LRA pós-contraste. ${ }^{13}$

Atualmente não existe evidência suficiente para recomendar hidratação oral como a única forma de profilaxia. ${ }^{13}$ Também não se encontra recomendada a administração de $\mathrm{N}$-acetilcisteina, medicação transitória com estatina em alta dose, inibidores da enzima de conversão da angiotensina, antagonistas dos receptores da angiotensina ou vitamina C, com o intuito hipotético de evitar LRA pós-contraste. ${ }^{13}$

Para reduzir o risco de LRA pós-contraste, é recomendado administrar a dose mínima necessária para o propósito, preferir contrastes com baixa ou normo-osmolalidade e utilizar uma razão $\frac{\text { dose de contraste em gramas }}{\text { TFG absoluta }}<1,1$ e uma

volume de contraste em $\mathrm{mL}$ razão inferior a 3 no contraste intra-arterial com primeira passagem renal. ${ }^{13}$

A metformina tem sido associada à ocorrência de acidose láctica. No entanto, a evidência mais recente indica que este evento é muito raro (3 - 9/100 000 doentes/ano) e acontece quase exclusivamente em doentes que têm alto risco para acidose láctica independentemente da administração de metformina. ${ }^{5,13}$ Apesar de ser um efeito secundário grave, potencialmente letal, precisa de um evento associado para acontecer, como por exemplo, falência respiratória, insuficiência renal, sépsis, hipoperfusão ou cirrose. ${ }^{8,11-13}$
O risco de utilização da metformina aquando da administração de contraste iodado prende-se com o facto de, caso haja LRA pós-contraste, o agravamento da função renal poder levar à diminuição da excreção renal de metformina, aumentando os seus níveis plasmáticos e podendo propiciar a ocorrência de acidose láctica.

Atualmente, não existe consenso em relação à melhor abordagem sobre a suspensão de metformina em pessoas com diabetes que vão ser submetidas à administração de contraste iodado. ${ }^{11,13,17}$ As recomendações que nos parecem ter maior evidência são as feitas pelas European Society of Urogenital Radiology, American College of Radiology e Sociedade Holandesa de Radiologia, baseadas em vários estudos e metanálises. ${ }^{13}$

Devido à falta de evidência publicada sobre a utilização de metformina em doentes submetidos a contraste iodado, as recomendações prévias eram baseadas em consensos e muito limitativas. ${ }^{13}$ À medida que se foi percebendo o baixo risco de acidose láctica, as recomendações têm sido cada vez menos restritivas. ${ }^{13,17}$

$\mathrm{Na}$ Tabela 4 apresentamos as recomendações sobre a suspensão de metformina em pessoas com diabetes sob esta terapêutica.

A TFGe deve ser avaliada nos sete dias antes da administração de contraste iodado em doentes com doença aguda, deterioração aguda de doença crónica ou em caso de internamento, e nos três meses antes em todos os outros doentes. ${ }^{13}$ A TFGe deve ser também avaliada 48 horas após a administração de contraste iodado, em doentes com TFGe inferior a $30 \mathrm{~mL} / \mathrm{min} / 1,73 \mathrm{~m}^{2}$ submetidos a contraste intravenoso ou contraste intra-arterial com segunda passagem real, e em todos os doentes submetidos a contraste iodado intra-arterial com primeira passagem renal independentemente da TFGe de base..$^{13,17}$

Nos doentes que estejam sob terapêutica de substituição da função renal (ex: diálise) não é necessário adaptar o momento de administração de contraste intravascular ao esquema de diálise. No entanto, isto pode ser feito para reduzir a sobrecarga de volume. ${ }^{13}$

Importa ainda referir que TFGe deve ser calculada nos adultos através da fórmula CKD-EPI e nas crianças através da fórmula de Schwartz revista. ${ }^{13}$

\section{Precauções em pessoas com diabetes submetidas a exames que necessitam de jejum}

O risco da realização de exames de imagem que necessitem de jejum merece particular atenção nos indivíduos

Tabela 3 - Tipo de profilaxia recomendada de acordo com o tipo de administração de contraste ${ }^{13}$

\begin{tabular}{ll}
\hline Tipo de contraste & Esquema de profilaxia \\
\hline Contraste intravenoso ou intra-arterial com & $3 \mathrm{~mL} / \mathrm{kg} / \mathrm{h}$ de bicarbonato a 1,4\% 1 hora antes administração contraste \\
segunda passagem renal & $1 \mathrm{~mL} / \mathrm{kg} / \mathrm{h}$ de cloreto de sodio a $0,9 \% 3-4$ horas antes da adminstração de \\
& contraste e $4-6$ horas após a administração de contraste \\
& $3 \mathrm{~mL} / \mathrm{kg} / \mathrm{h}$ de bicarbonato a $1,4 \% 1$ hora antes administração contraste e $1 \mathrm{~mL} / \mathrm{kg} / \mathrm{h}$ \\
Contraste intra-arterial com primeira & $4-6$ horas após a administração de contraste \\
passagem renal & $1 \mathrm{~mL} / \mathrm{kg} / \mathrm{h}$ de cloreto de sodio a 0,9\% $3-4$ horas antes da adminstração de \\
& contraste e $4-6$ horas após a administração de contraste \\
\hline
\end{tabular}


Tabela 4 - Recomendações sobre a suspensão de metformina das diferentes sociedades médicas ${ }^{11,13,17}$

\begin{tabular}{|c|c|c|}
\hline TFGe & Tipo de contraste & Recomendações \\
\hline \multicolumn{3}{|l|}{ ESUR, ACR, SHR } \\
\hline$>30 \mathrm{~mL} / \mathrm{min} / 1,73 \mathrm{~m}^{2}$ sem evidência de LRA & IV ou IA com $2^{\mathrm{a}}$ passagem renal & Manter administração \\
\hline$<30 \mathrm{~mL} / \mathrm{min} / 1,73 \mathrm{~m}^{2}$ & IV ou IA com $2^{a}$ passagem renal & \multirow{3}{*}{$\begin{array}{l}\text { Parar metformina } \\
\text { Reavaliar TFGe } 48 \text { horas após a administração } \\
\text { de contraste iodado } \\
\text { Reiniciar metformina se TFGe estável }\end{array}$} \\
\hline---- & IA com $1^{\text {a }}$ passagem renal & \\
\hline Com evidência de LRA & - & \\
\hline \multicolumn{3}{|l|}{ SSUR } \\
\hline$>45 \mathrm{~mL} / \mathrm{min} / 1,73 \mathrm{~m}^{2}$ & IV ou IA com $2^{\mathrm{a}}$ passagem renal & Manter administração \\
\hline$<45 \mathrm{~mL} / \mathrm{min} / 1,73 \mathrm{~m}^{2}$ & IV ou IA com $2^{\mathrm{a}}$ passagem renal & \multirow{4}{*}{$\begin{array}{l}\text { Parar metformina } \\
\text { Reavaliar TFGe } 48 \text { horas } \\
\text { Reiniciar metformina se TFGe estável }\end{array}$} \\
\hline Razão dose contraste (g)/TFGe $\geq 1$ & IV ou IA com $2^{a}$ passagem renal & \\
\hline LRA ou risco de LRA & ----- & \\
\hline---- & IA com $1^{\text {a }}$ passagem renal & \\
\hline \multicolumn{3}{|l|}{ ADA } \\
\hline $30-60 \mathrm{~mL} / \mathrm{min} / 1,73 \mathrm{~m}^{3}$ & ---------------- & Deve ser temporariamente suspensa \\
\hline
\end{tabular}

ESUR: European Society of Urogenital Radiology; ACR: American College of Radiology; SHR: Sociedade Holandesa de Radiologia; IV: intravenoso; IA: intra-arterial; SSUR: Sociedade Sueca de Radiologia Urogenital; ADA: American Diabetes Association

sob insulinoterapia e anti-diabéticos orais com risco de hipoglicémia. ${ }^{8,19}$ Durante o período de jejum num indivíduo sem diabetes, a ligeira redução inicial da glicemia leva à diminuição da produção pancreática de insulina, a um aumento da produção de glucagon e à resposta catecolaminérgica, o que permite a manutenção da glicemia. ${ }^{20}$ Numa pessoa com diabetes insulinotratada, a insulina encontra-se já em circulação e, dependendo do caso, o pâncreas pode não ter capacidade para adaptar a secreção de glucagon. ${ }^{20}$ Desta forma, a pessoa com diabetes está frequentemente dependente apenas da resposta catecolaminérgica que também pode estar limitada pela presença de neuropatia autonómica ou hipoglicemias recorrentes. ${ }^{20}$

As insulinas de ação curta ou rápida são as que se encontram associadas ao maior risco de hipoglicemia, já que ao mimetizar a secreção de insulina associada à refeição têm um rápido e marcado pico de ação. ${ }^{8}$

A pessoa com diabetes sob anti-diabéticos orais com risco de hipoglicemia - sulfonilureias (glibenclamida, gliclazida, glimepirida e glipizida) e glinidas (nateglinida) - tem também maior risco de hipoglicemia durante o jejum, sendo recomendado que estes doentes adiem a toma do fármaco até que haja ingestão de alimentos, após o exame. ${ }^{8,19}$

Assim, para minimizar o risco de hipoglicemia, os exames dos doentes insulinotratados que obriguem a jejum devem ser marcados para o início da manhã, de forma a reduzir as alterações necessárias ao esquema de insulina. Em geral, este procedimento evita a necessidade de alteração da insulina basal, sendo recomendável o adiamento da administração de insulina de ação curta ou rápida até à ingestão de alimentos após o término do exame.

$\mathrm{Na}$ impossibilidade destes exames serem realizados no início da manhã, não deve ser administrada a insulina de ação curta ou rápida para as refeições omitidas. Se o doente estiver marcado para a tarde, em geral, poderá ingerir o pequeno-almoço e ser tratado com a insulina habitual dessa refeição. Relativamente à insulina basal, é importante que os indivíduos com diabetes tipo 1 mantenham a sua insulina basal, embora na maioria das situações um eventual atraso na administração deste tipo de insulina (de uma a três horas) não tenha consequências relevantes. De igual modo, atrasos superiores aos indicados na administração da insulina degludec são tolerados sem descontrolo glicémico relevante..$^{21,22}$

Apesar da manutenção da insulina basal parecer ser segura, a dose de insulina basal glargina ou detemir da manhã poderá ser reduzida entre $20 \%$ a $25 \%$, dependendo do nível de controlo glicémico de cada doente, do seu risco de hipoglicemia e do tempo de jejum requerido. ${ }^{22,23}$ Relativamente à insulina $\mathrm{NPH}$, poderão considerar-se necessárias reduções na ordem dos $50 \%$ nessa manhã, em função do seu perfil com pico de maior ação por volta das quatro horas. ${ }^{23}$ Doentes com hiperglicemia recente não devem, na maioria dos casos, sofrer reduções na dose de insulina basal.

No caso de prolongamentos imprevistos do jejum, em que tenha sido administrada a dose habitual de insulina basal, recomenda-se intensificar a vigilância por glicemia capilar e, dependendo do nível de glicemia e tempo estimado para concluir o jejum, pode iniciar-se uma perfusão de dextrose a $5 \% .{ }^{24}$ Muitas instituições têm protocolos sobre controlo da diabetes durante procedimentos cirúrgicos e peri-operatório, sendo razoável aplicar aos exames de imagiologia uma conduta semelhante aos protocolos para cirurgia minor. ${ }^{23,24}$

A terapêutica com sulfonilureias deve em geral ser omitida na manhã em que o doente permanecerá em jejum dependendo de controlo glicémico e com vigilância glicémica reforçada nesse período. ${ }^{23}$

Durante o período de jejum, a pessoa com diabetes tratada com fármacos com risco de hipoglicemia deve vigiar a glicemia a cada quatro a seis horas, e sempre que clinicamente recomendado. ${ }^{24}$ 


\section{PET-FDG}

A PET-FDG é um exame realizado frequentemente na prática clínica, tanto para estadiamento ou vigilância de patologia oncológica, como para estudo de patologia neurológica, inflamatória ou infeciosa. ${ }^{25}$

A estrutura química da FDG é semelhante à da glicose natural pelo que, na presença de hiperglicemia, há competição direta entre a captação celular de glicose plasmática e da FDG..$^{25}$ Esta redução da captação da FDG pode levar a redução da sensibilidade do exame, que parece acontecer apenas para glicemias capilares superiores a $200 \mathrm{mg} / \mathrm{dL} .^{25}$ Por isso, com o objetivo de não comprometer a sensibilidade do exame, recomenda-se que a glicemia capilar seja inferior a esse valor antes da administração de FDG..$^{25}$

Caso o exame seja realizado para estudo de doença neurológica ou lesão no sistema nervoso central, a glicemia capilar deve ser inferior a $160 \mathrm{mg} / \mathrm{dL}$, pois para valores superiores a estes o contraste entre a substância cinzenta e a substância branca pode ser perdido, para além de que o aumento do ruído pode reduzir a sensibilidade do exame. ${ }^{25}$

A metformina pode, também, alterar a sensibilidade da PET-FDG, uma vez que aumenta a acumulação da FDG a nível intestinal, particularmente no cólon. ${ }^{25}$ Este aumento de captação provocado pelo fármaco pode levar a diminuição da sensibilidade do exame e a um aumento de falsos negativos no estudo de neoplasias abdominais e pélvicas. ${ }^{25}$ Não existem recomendações sobre como atuar em doentes sob terapêutica com metformina que vão realizar PET-FDG, nem quando esta deva ser suspensa caso o objetivo do exame seja avaliar lesões abdominais. ${ }^{25} \mathrm{O}$ risco de diminuição da sensibilidade do exame tem de ser considerado caso a caso e equilibrado com o risco de hiperglicemia associado à suspensão da terapêutica. ${ }^{25}$ Caso se opte pela suspensão, há alguma evidência de que deverá ter uma duração mínima de 72 horas. ${ }^{25}$

Ao alterar a biodistribuição da FDG, a terapêutica com insulina também influencia a sensibilidade deste exame. ${ }^{25} \mathrm{~A}$ insulina de ação curta ou rápida não deve ser administrada nas quatro a seis horas antes, e a insulina basal deverá ser administrada apenas após a administração da FDG e a realização da PET. ${ }^{25}$ Não existem atualmente recomendações sobre o período de tempo entre a administração das novas insulinas de ação ultra-rápida (insulinas aspártica ultra-rápida e lispro-aabc). No entanto, de acordo com a sua farmacocinética e até que existam recomendações específicas, recomendamos um procedimento semelhante ao conselhado para as insulinas clássicas de ação curta ou rápida. ${ }^{26,27} \mathrm{Em}$ indivíduos sob terapêutica com perfusão subcutânea contínua de insulina recomenda-se suspensão da infusão pelo menos quatro horas antes da administração da FDG. ${ }^{25}$

Não se encontra ainda bem determinada a influência dos inibidores de SGLT2 na avaliação renal por PET-18FDG. ${ }^{28}$ Sabe-se que estes medicamentos podem acelerar o trânsito de glicose renal, parâmetro que é importante na avaliação da função renal por PET-FDG. ${ }^{28}$

Atualmente, não existe evidência sobre a influência de outros fármacos anti-diabéticos na sensibilidade da PET-18FDG, à excepção da pioglitazona, que parece aumentar a captação de FDG pelas lesões malignas. ${ }^{29}$

\section{Proposta de protocolo}

Após revisão da literatura, criámos uma proposta de protocolo de atuação para as pessoas com diabetes que vão realizar exames de imagem.

$\mathrm{Na}$ Tabela 5 apresentam-se as medidas gerais de prevenção de LRA associada à administração de contraste.

Tabela 5 - Medidas gerais recomendadas na prevenção de LRA associada ao contraste

\begin{tabular}{l} 
Medidas gerais na prevenção de LRA associada ao contraste: \\
\hline Suspender, sempre que possível, os fármacos potencialmente nefrotóxicos \\
Fazer profilaxia com hidratação endovenosa se fatores de risco para LRA pós-contraste \\
Administrar a dose mínima de contraste iodado necessária para o propósito \\
Preferir contrastes de baixa ou normo-osmolalidade \\
Se utilização de contraste intra-arterial com primeira passagem renal: \\
Razão $\frac{\text { dose de contraste em gramas }}{\text { TFG absoluta }}<1,1$ e \\
Razão $\frac{\text { volume de contraste em } \mathrm{mL}}{\text { TFGe }}<3$
\end{tabular}

Tabela 6 - Protocolo sobre administração de contraste iodado em pessoas medicadas com metformina

\begin{tabular}{lll}
\hline TFGe & Tipo de contraste & Recomendações \\
\hline$>30 \mathrm{~mL} / \mathrm{min} / 1,73 \mathrm{~m}^{2}$ sem evidência de LRA & IV ou IA com 2 ${ }^{\text {a }}$ passagem renal & Manter administração \\
$<30 \mathrm{~mL} / \mathrm{min} / 1,73 \mathrm{~m}^{2}$ & IV ou IA com 2 ${ }^{\text {a }}$ passagem renal & Parar metformina \\
---- & IA com 1ª passagem renal & Reavaliar TFGe 48 horas \\
Com evidência de LRA & -------- & Reiniciar metformina se TFGe estável e \\
\hline
\end{tabular}


Tabela 7 - Protocolo de atuação em pessoas com diabetes insulinotratadas para exames que impliquem jejum

Recomendações para exames que impliquem jejum em doentes insulinotratados:
Marcar o exame para o inicio da manhã
Não administrar insulina de ação curta ou rápida da refeição que se omite
Na refeição após o exame administrar insulina de ação curta ou rápida de acordo com o esquema para aquela refeição
Glicemia deve ser avaliada ocasionalmente e sempre que clinicamente justificável
Contactar o médico assistente para avaliar a eventual necessidade de ajuste da insulina basal

Tabela 8 - Recomendações na pessoa com diabetes que vai ser submetida a PET-FDG

$\begin{array}{ll}\text { Objetivo glicémico } & \text { Geral }<200 \mathrm{mg} / \mathrm{dL} \\ & \text { Estudo de doença neurológica ou lesão do } \mathrm{SNC}<160 \mathrm{mg} / \mathrm{dL} \\ \text { Metformina } & \text { Avaliação da necessidade de suspensão pelo médico assistente tendo em consideração: } \\ & \text { - Diminuição da sensibilidade para estudo de lesões abdominais e pélvicas } \\ & \text { - Risco de hiperglicemia associada à suspensão da terapêutica } \\ & \text { Caso se opte por suspender fazê-lo no mínimo } 72 \text { horas antes do exame }\end{array}$

Insulina

Não administrar insulina de ação curta ou rápida nas $4-6$ horas antes da administração de FDG Administrar Insulina basal após a realização do exame

Se PSCI deve-se suspender a infusão 4 horas antes da administração de FDG

PSCI: perfusão subcutânea contínua de insulina

Há várias situações sem consenso na literatura sobre a melhor abordagem. Nestas tentámos identificar as que apresentam maior evidência científica.

Na Tabela 6 apresentamos o protocolo de atuação nos doentes submetidos a contraste iodado e que estejam sob terapêutica com metformina.

Optámos por favorecer as recomendações das European Society of Urogenital Radiology, American College of Radiology e Sociedade Holandesa de Radiologia, baseadas em vários estudos e metanálises, uma vez que são aquelas que apresentam melhor qualidade de informação. ${ }^{13}$

Em relação à realização de exames que impliquem jejum em indivíduos tratados com insulinoterapia, as nossas recomendações encontram-se na Tabela 7.

Para os indivíduos que vão ser submetidos a PET-FDG, as recomendações encontram-se na Tabela 8.

\section{CONCLUSÃO}

A gestão do indivíduo com diabetes é complexa. $O$ risco de acidose láctica no indivíduo com diabetes sob terapêutica com metformina submetido a contraste iodado não parece ser tão elevado como se pensava inicialmente. Desta forma, as indicações para suspensão temporária de metformina são atualmente menos restritivas. Os exames que obriguem a jejum nos indivíduos sob insulinoterapia devem ser marcados para o início da manhã, de forma a serem necessárias menos alterações terapêuticas. $\mathrm{Na}$ maior parte dos casos poderá apenas ser recomendado adiar a administração de insulina de ação curta ou rápida para o momento da ingestão de alimentos, após o exame. Na PET-FDG, tanto a terapêutica (com metformina ou insulina) como a hiperglicemia podem diminuir a sensibilidade do exame. Em relação à terapêutica com metformina, a suspensão deve ser ponderada caso a caso, pelo risco de hiperglicemia associado. Em relação à terapêutica com insulina, as recomendações dependem do tipo de insulina, estando recomendado não administrar insulina de ação curta ou rápida nas quatro horas antes da administração de FDG, e administrar insulina basal após a realização do exame.

Com esta proposta de protocolo pretendemos resumir e simplificar a orientação dos indivíduos com diabetes que vão ser submetidos a exames de imagem.

\section{CONFIDENCIALIDADE DOS DADOS}

Os autores declaram ter seguido os protocolos de confidencialidade de dados na realização da presente investigação.

\section{CONFLITOS DE INTERESSE}

Os autores declaram não haver conflito de interesses na realização da presente investigação.

\section{FONTES DE FINANCIAMENTO}

Não existiram fontes externas de financiamento para a presente investigação.

\section{REFERÊNCIAS}

1. World Health Organization. Diabetes 2020. [consultado 2020 dez 04]. Disponível em: https://www.who.int/news-room/fact-sheets/detail/ diabetes.

2. Raposo J. Diabetes: factos e números 2016, 2017 e 2018. Rev Port Diabetes. 2020;15:19-27.

3. Ciarrapico A, Ugenti R, Di Minco L, Santori E, Altobelli S, Coco I, et al. Diagnostic imaging and spending review: extreme problems call for extreme measures. Radiol Med. 2017;122:288-93.

4. Barsanti C, Lenzarini F, Kusmic C. Diagnostic and prognostic utility of non-invasive imaging in diabetes management. World J Diabetes. 2015;6:792-806.

5. Riddle M, Ahmann A. Therapeutics of type 2 diabetes mellitus. In: Melmed S, Koenig R, Rosen C, Auchus R, Goldfin A, editors. Williams Textbook of Endocrinology. $14^{\text {th }}$ ed. Amsterdam: Elsevier; 2019. p.1371402.

6. American Diabetes Association. Classification and diagnosis of 
diabetes: standards of medical care in Diabetes-2021. Diabetes Care. 2021;44:S34-9.

7. Mobasseri M, Shirmohammadi M, Amiri T, Vahed $N$, Hosseini Fard $H$, Ghojazadeh M. Prevalence and incidence of type 1 diabetes in the world: a systematic review and meta-analysis. Health Promot Perspect. 2020;10:98-115.

8. American Diabetes Association. Pharmacologic approaches to glycemic treatment: standards of medical care in diabetes-2020. Diabetes Care. 2020;43:S98-s110.

9. Almeida MC, Dores J, Ruas L. Consenso "Diabetes Gestacional": atualização 2017. Rev Port Diabetes. 2017;12:24-38.

10. McCartney CR, Marshall JC. Clinical practice. Polycystic ovary syndrome. N Engl J Med. 2016;375:54-64.

11. American Diabetes Association. Microvascular complications and foot care: standards of medical care in diabetes-2020. Diabetes Care. 2020;43:S135-51

12. Yang S, Dai Y, Liu Z, Wang C, Meng Q, Huo X, et al. Involvement of organic cation transporter 2 in the metformin-associated increased lactate levels caused by contrast-induced nephropathy. Biomed Pharmacother. 2018;106:1760-6.

13. van der Molen A, Reimer P, Dekkers I, Bongartz G, Bellin MF, Bertolotto $\mathrm{M}$, et al. Post-contrast acute kidney injury. Part 2: risk stratification, role of hydration and other prophylactic measures, patients taking metformin and chronic dialysis patients: Recommendations for updated ESUR Contrast Medium Safety Committee guidelines. Eur Radiol. 2018;28:2856-69.

14. Hiremath S, Kayibanda JF, Chow B, Fergusson D, Knoll G, Shabana $W$, et al. Drug discontinuation before contrast procedures and the effect on acute kidney injury and other clinical outcomes: a systematic review protocol. Syst Rev. 2018;7:34

15. American College of Radiology Committee on Drugs and Contras Media. ACR Manual on Contrast Media. Philadelphia: ACR; 2021.

16. Organisation for Economic Co-operation and Development. Computed tomography (CT) exams: OECD; 2018. [consultado 2020 dez 04] Disponível em: https://data.oecd.org/healthcare/computed-tomographyct-exams.htm.

17. Nyman U, Ahlkvist J, Aspelin P, Brismar T, Frid A, Hellström M, et al. Preventing contrast medium-induced acute kidney injury: side-by-side comparison of Swedish-ESUR guidelines. Eur Radiol. 2018;28:5384-95.
18. Vlachopanos G, Schizas D, Hasemaki N Georgalis A. Pathophysiology of contrast-induced acute kidney injury (CIAKI). Curr Pharm Des. 2019;25:4642-7.

19. Infarmed. Prontuário Terapêutico 2020. [consultado 2020 dez 4]. Disponível em: https://app10.infarmed.pt/prontuario/index.php.

20. Al-Arouj M, Assaad-Khalil S, Buse J, Fahdil I, Fahmy M, Hafez S, et al. Recommendations for management of diabetes during ramadan. Diabetes Care. 2005;28:2305-11.

21. Mathieu C, Hollander P, Miranda-Palma B, Cooper J, Franek E, RussellJones $D$, et al. Efficacy and safety of insulin degludec in a flexible dosing regimen vs insulin glargine in patients with type 1 diabetes (BEGIN: Flex T1): a 26-week randomized, treat-to-target trial with a 26-week extension. J Clin Endocrinol Metab. 2013;98:1154-62.

22. Mucha G, Merkel S, Thomas W, Bantle J. Fasting and insulin glargine in individuals with type 1 diabetes. Diabetes Care. 2004;27:1209-10.

23. American Diabetes Association. 15. Diabetes care in the hospital: standards of medical care in diabetes-2021. Diabetes Care. 2021;44:S211-20.

24. Umpierrez G, Hellman R, Korytkowski M, Kosiborod M, Maynard G, Montori $\mathrm{V}$, et al. Management of hyperglycemia in hospitalized patients in non-critical care setting: an endocrine society clinical practice guideline. J Clin Endocrinol Metab. 2012;97:16-38.

25. Finessi M, Bisi G, Deandreis D. Hyperglycemia and 18F-FDG PET/ $\mathrm{CT}$, issues and problem solving: a literature review. Acta Diabetol. 2020;57:253-62.

26. Donner T, Sarkar S. Insulin - pharmacology, therapeutic regimens, and principles of intensive insulin therapy. Endotext. [consultado $2021 \mathrm{fev}$ 17] Disponível em: https://www.ncbi.nlm.nih.gov/books/NBK278938/.

27. European Medicines Agency, 2020. Assessment report Liumjev [consultado 2021 fev 17] Disponível em: https://www.ema.europa.eu/ en/documents/assessment-report/liumjev-epar-public-assessmentreport en.pdf.

28. Rasul S, Geist B, Brath H, Baltzer P, Sundar L, Pichler V, et al. Response evaluation of SGLT2 inhibitor therapy in patients with type 2 diabetes mellitus using (18)F-FDG PET/MRI. BMJ Open Diabetes Res Care. 2020;8:e001135.

29. Evangelista L, Gori S, Rubini G, Gallo M. Management of hyperglycemia in oncological patients scheduled for an FDG-PET/CT examination. Clin Transl Imaging. 2019;7:447-50. 